Cahiers $d u$ MONDE RUSSE

\section{Cahiers du monde russe}

Russie - Empire russe - Union soviétique et États indépendants

$48 / 1 \mid 2007$

Émigrations au début du XXe siècle

\title{
L'Association des Étudiants russes de Paris
}

\section{Boris Czerny}

\section{OpenEdition \\ Journals}

Édition électronique

URL : https://journals.openedition.org/monderusse/8983

DOI : 10.4000/monderusse.8983

ISSN : $1777-5388$

Éditeur

Éditions de l'EHESS

Édition imprimée

Date de publication : 15 janvier 2007

Pagination : $5-21$

ISBN : 978-2-7132-2146-0

ISSN : $1252-6576$

\section{Référence électronique}

Boris Czerny, "L'Association des Étudiants russes de Paris », Cahiers du monde russe [En ligne], 48/1 | 2007, mis en ligne le 01 janvier 2007, consulté le 02 septembre 2022. URL : http://

journals.openedition.org/monderusse/8983; DOI : https://doi.org/10.4000/monderusse.8983 
chercher : repérer : avancer

Cet article est disponible en ligne à l'adresse :

http://www.cairn.info/article.php?ID REVUE=CMR\&ID NUMPUBLIE=CMR 481\&ID ARTICLE=CMR 4810005

\title{
L'Association des Étudiants russes de Paris
}

\section{par Boris CZERNY}

\section{Editions de l'EHESS | Cahiers du monde russe}

\author{
2007/1 - Vol 48 \\ ISSN 1252-6576 | ISBN 9782713221460 | pages 5 à 21
}

Pour citer cet article :

-Czerny B., L’Association des Étudiants russes de Paris, Cahiers du monde russe 2007/ 1, Vol 48, p. 5-21.

Distribution électronique Cairn pour les Editions de l'EHESS.

(C) Editions de l'EHESS. Tous droits réservés pour tous pays.

La reproduction ou représentation de cet article, notamment par photocopie, n'est autorisée que dans les limites des conditions générales d'utilisation du site ou, le cas échéant, des conditions générales de la licence souscrite par votre établissement. Toute autre reproduction ou représentation, en tout ou partie, sous quelque forme et de quelque manière que ce soit, est interdite sauf accord préalable et écrit de l'éditeur, en dehors des cas prévus par la législation en vigueur en France. Il est précisé que son stockage dans une base de données est également interdit. 


\section{L’ASSOCIATION DES ÉTUDIANTS RUSSES DE PARIS}

Les archives de l'Association des étudiants russes à Paris (Obščestvo russkih studentov $v$ Pariže, ci-après AER) ${ }^{1}$ et les fonds Bebutov ${ }^{2}$, conservés à la Bibliothèque de documentation internationale contemporaine (BDIC), apportent un éclairage original et inédit sur l'émigration estudiantine russe au début du $\mathrm{XX}^{\mathrm{e}}$ siècle. Tous deux témoignent en effet de l'émergence d'une migration organisée, dans laquelle les associations estudiantines à l'étranger jouent un rôle important de relais d'information et d'accueil. En ces temps d'une pratique migratoire en pleine expansion, les correspondances adressées au monde associatif révèlent les attitudes et les motivations des futurs étudiants. On voit alors apparaître dans la décennie d'avant-guerre la relative banalisation des départs de Russie en vue d'acquérir une formation supérieure à l'étranger. Bien que motifs politiques et discriminations nationales, en particulier l'antisémitisme, demeurent des facteurs essentiels, la migration se présente désormais comme une éventualité stratégique, sinon généralisée du moins envisagée, pour un nombre croissant de jeunes. Leur voix est celle de la foule anonyme des étudiants russes souvent mis en scène comme des personnages «romantiques » dans les mémoires d'écrivains et d'hommes politiques ${ }^{3}$. Il n'est pas question ici de ces jeunes

1. F delta res 815 .

2. David Osipovič (Iosifovič) Bebutov (1859-1916) était le petit-fils d'un ancien gouverneur de Tiflis, Vasilij Ašhar-Beka, qui se signala par ses exploits militaires lors de la guerre de Crimée. Au début du $\mathrm{XX}^{\mathrm{e}}$ siècle, Bebutov résidait à Saint-Pétersbourg, où il fréquentait assidûment les cercles maçonniques. Il collecta de nombreux documents relatifs aux partis révolutionnaires et à la social-démocratie en particulier. Après la révolution d'Octobre, les archives Bebutov furent transférées en Allemagne, où elles furent remises aux responsables de la social-démocratie allemande. À l'arrivée des nazis au pouvoir, les documents rassemblés par Bebutov furent dispersés entre l'Angleterre, Amsterdam et Paris. Après la Seconde Guerre mondiale, Anatole de Monzie apprit l'existence de ces archives et en fit l'acquisition. Par la suite, elles furent transférées à la BDIC, où elles se trouvent aujourd'hui sous la cote F delta res 811 .

3. La liste est longue. Citons, entre autres : E. Lundberg, Zapiski pisatelja [Notes d'un écrivain], vol. II : 1920-1924, L.: Pisatelej, 1938; Ossip Piatnitsky, Souvenirs d'un bolchevik, 1896-1917, P. : Bureau d'édition, 1931 ; V.Černov, Pered burej [Avant la tempête], New 
dont le « regard fiévreux » trahissait un sentiment de haine envers le régime tsariste. Les jeunes filles n'ont pas grand-chose en commun avec ces héroïnes « de feu » qui furent l'archétype de l'étudiante russe (juive russe) dans la littérature française du début du $\mathrm{XX}^{\mathrm{e}}$ siècle ${ }^{4}$. Les documents à caractère administratif et logistique dont nous disposons (lettres diverses, liste de menus, comptes rendus de réunions, etc.) brossent le portrait d'étudiants « ordinaires », mus surtout par une volonté d'ascension sociale par le biais d'une formation supérieure. Au travers de ces correspondances, nous pouvons suivre les parcours des candidats au départ, depuis leur projet d'étudier à l'étranger jusqu'à leur installation à Paris ou dans une autre ville universitaire en Occident, en passant par leur participation à la vie associative russe. Contrairement aux associations d'Allemagne et de Suisse, le fonctionnement des associations d'étudiants russes en France n'a encore pas fait l'objet d'études. Par la présentation du fonds de l'AER, nous espérons contribuer à combler cette lacune.

\section{Les canaux d'information}

Dans une lettre adressée en 1908 à des journaux parisiens, les dirigeants de l'AER annoncent la tenue d'un bal à l'occasion du vingt-cinquième anniversaire de leur association ${ }^{5}$. L'AER est une des plus anciennes organisations d'étudiants russes à l'étranger, bien que les principales études sur le sujet ne la mentionnent pas 6 . Entre 1908 et 1913, ses locaux sont situés 5, rue Malebranche dans le 5e arrondissement de Paris. Par la suite, l'AER s'installe 3, rue du Puits-de-l'Ermite, dans le 13e. Elle reste ainsi sur la rive gauche de la Seine, dans le « quartier russe » circonscrit par les $5^{\mathrm{e}}, 6^{\mathrm{e}}$ et $13^{\mathrm{e}}$ arrondissements.

York : Izd. imeni Čehova, 1953 ; Mark Višnjak, Dan' prošlomu [L'hommage au passé], New York : Izd. imeni Čehova, 1954 ; id., Gody emigracii, Pariž-Nju-Jork (Vospominanija) [Mes années dans l'émigration, Paris-New York (souvenirs)], Stanford : Hoover Institution Press, 1970 ; Boris Pasternak, Sauf-conduit, P.: Buchet-Chastel, 1959 ; Ilya Ehrenbourg, Les Années et les hommes, P.: Gallimard, 1962 ; Naum Goldman, Autobiographie, P.: Fayard, 1971 ; Nadejda Mandelstam, Contre tout espoir, P.: Gallimard, 1972 ; Angelica Balabanoff, Ma vie de rebelle, P.: Balland, 1981 ; Nina Berberova, C'est moi qui souligne, Arles : Actes Sud, 1989.

4. Voir, par exemple, Georges Duhamel, La Pierre d'Horeb et Biographie de mes fantômes.

5. F delta res 815 (4), F delta res 811 (8) (12). L'AER a été fondée en 1883.

6. Voir, entre autres : A. Ivanov, Studenčestvo Rossii konca 19 - načala 20 veka : social'noistoričeskaja sud'ba, [Les étudiants en Russie à la fin du XIX et au début du XX $\mathrm{X}^{\mathrm{e}}$ siècle : destin historique et social], M. : ROSSPEN, 1999 ; Ja. Ščapov, « Russkie studenty v zapadnoevropejskoj vyščej škole v načale 20 veka » [Les étudiants russes dans les établissements d'enseignement supérieur en Occident au début du XX ${ }^{\mathrm{e}}$ siècle], Istoričeskie zapiski, Moscou, $\mathrm{n}^{\circ}$ 15, 1987 ; Claudie Weill, Étudiants russes en Allemagne: 1910-1914. Quand la Russie frappait aux portes de l'Europe, P. : l'Harmattan, 1996; Victor Karady, Relations inter-universitaires et rapports culturels en Europe (1871-1945). Rapport de fin d'études, P. : Centre de sociologie de l'éducation de la culture, 1992 ; Natalie Tikhonov, La Quête du savoir : Étudiantes de l'Empire russe dans les universités suisses (1864-1920), Thèse pour obtenir le grade de docteur de l'université de Genève/ de docteur de l'EHESS, 3 vol., Genève, 2004 ; Dmitri Gouzevitch, Irina Gouzevitch, «Les contacts franco-russes dans le monde de l'enseignement supérieur technique et de l'art de l'ingénieur », Cahiers du Monde russe et soviétique, 34 (3), 1993, p. 345-368. Certains ouvrages concernant les étudiants russes juifs sont cités à la note 9 . 
L'importance croissante de l'AER s'explique aisément par le durcissement du climat politique après 1905 qui se traduit notamment par la réintroduction d'un numerus clausus pour les juifs et l'interdiction faite aux femmes d'accéder à l'université, édictés par une nouvelle loi. Pour obtenir des informations fiables sur les enseignements dispensés et les conditions de vie en Europe de l'Ouest, un certain nombre se tourne vers les associations d'étudiants russes à l'étranger. Ces dernières s'avèrent l'une des principales sources de renseignements.

Sur les lettres ${ }^{7}$ conservées dans les archives de l'AER, les adresses des expéditeurs sont clairement indiquées. Elles proviennent principalement de l'Ouest de l'Empire comprenant la zone de résidence, puis de la Russie proprement dite et, pour un petit nombre, du Caucase et des régions limitrophes. Ces trois groupes dessinent les zones dans lesquelles l'AER est connue.

$\mathrm{Si}$, le plus souvent, les lettres sont le fait d'une initiative individuelle, un nombre non négligeable d'entre elles sont rédigées par des groupes de deux à trois lycéens qui préfèrent affronter l'inconnu à plusieurs. Dans ces courriers, la palette des renseignements demandés se fait plus variée. Ainsi dans une carte postale expédiée de Tiflis, un étudiant, Viktor Nikolaevič Panov, demande des précisions sur les études d'agronomie, de médecine et, de manière générale, dans le domaine scientifique. De toute évidence, ces questions émanent de quatre personnes différentes ${ }^{8}$.

Les futurs étudiants réunissent leurs demandes dans des lettres collectives souvent pour des raisons d'économie, les services de l'AER étant en effet payants. L'association tarife vingt-cinq francs la traduction de documents officiels et la légalisation des diplômes. De plus, elle ne fournit de renseignements qu'aux personnes s'engageant à devenir membres de l'AER, moyennant cotisation. Enfin, seules les lettres munies d'un timbre-réponse de dix à vingt kopeks sont prises en considération (cinquante centimes pour les lettres envoyées de France et pour les réponses en France).

Nous pouvons supposer que, dans nombre de cas, la volonté qui animait les étudiants s'adressant à l'AER est restée de l'ordre de l'intention. Certains sont demeurés en Russie dans l'attente de nouvelles d'amis partis explorer une voie que d'autres emprunteront à leur suite. C'est ainsi que se cimente dans certaines villes occidentales la cohésion des « colonies » d'étudiants de Russie. Dans de nombreux domaines d'études, si ces jeunes gens jettent leur dévolu sur telle ou telle ville Toulouse et Nancy, par exemple ${ }^{9}$ - les raisons de leur choix sont rarement

7. Au nombre de 136 .

8. F delta res 815 (13).

9. Pour Toulouse, voir Colette Zytnicki, «D'Odessa à Toulouse. L'émigration juive russe à Toulouse dans la première moitié du XX siècle », in Juifs de Russie et émigrés en France, Moscou, Jérusalem : Mosty kul’tury-Gešarim, 2000,p. 175 ; pour Nancy, voir : P. Buhara, « Političeskaja aktivnost' evrejskih studentov iz Vostočnoj Evropy v Nansi » [L'Activité politique des étudiants juifs d'Europe de l'Est à Nancy] », in Russkie evrei vo Francii : stat'i, publikacii, memuary i esse [Les juifs russes en France : articles, publications, mémoires et essais], Kn. 2, Jérusalem, 2002, p. 46-54; Dmitri Gouzevitch, Irina Gouzevitch, « Les étudiants de l'Europe de l'Est à l'Institut électrotechnique de Nancy », in Françoise Birck, André Grelon, éds., Un siècle de formation des ingénieurs électriciens: ancrage local et dynamique européenne, l'exemple de Nancy, P.: Éditions de la Maison des sciences de l'homme, 2006, etc. 
explicitées. Toutefois, la lettre de l'étudiant Petrovič Gladkov permet de comprendre, à travers son point de vue certes partiel mais intéressant, les motifs qui le poussent vers Nancy. Il écrit depuis la région du Don ${ }^{10}$ pour s'informer sur les établissements d'enseignement supérieur nancéiens car « les relations entre enseignants et étudiants y sont cordiales ». C'est là un exemple type de réputation de bouche à oreille qui joue en faveur d'une ville ou d'une université au détriment d'autres. C'est aussi le cas pour Grigorij Egol'skij, originaire de Novo-Ukrajnka dans le gouvernement de Herson, qui demande confirmation de renseignements qu'il tient d'un ami juriste ayant étudié en France ${ }^{11}$.

De retour au pays, d'aucuns peuvent mettre à profit leur expérience pour rédiger des guides. Ainsi un certain G. I. Gurevič ${ }^{12}$ de Minsk se réfère aux « célèbres » guides de Dmitrij Margolin13. Un étudiant, Moisej Markovič Brajnos, informe l'Association qu'il est l'auteur d'un ouvrage comportant « des renseignements complets et précis pour tous ceux qui souhaitent entrer dans des établissements d'enseignement supérieur et sur les conditions de vie en France ${ }^{14}$.

Les candidats au départ disposent aussi de la documentation fournie par les établissements d'enseignement supérieur français.

Les programmes d'études adressés à l'AER sont affichés dans les locaux de la rue Malebranche. Des exemplaires en étaient expédiés à des libraires en Russie, qui jouent alors le rôle d'intermédiaire dans cette diffusion de l'information. Ainsi P. Vasil'evskij, propriétaire d'une librairie à Taganrog, demande à l'AER de lui fournir des renseignements et une documentation sur l'Association des anciens élèves des écoles nationales des arts et métiers et sur l'Union des associations des écoles supérieures de commerce et de le mettre en relation avec d'autres associations d'étudiants russes à Vienne, Berlin et Leipzig. Il demande aussi à l'AER de signaler dans la presse l'existence de son magasin et sa fonction de relais ${ }^{15}$. Il est fort probable que de nombreuses autres librairies en Russie ont eu la même utilité.

Les journaux contribuent également à cette diffusion. Le relevé des comptes de l'AER pour l'année 1910 (de janvier à décembre) impute plus de $6 \%$ des dépenses aux frais de poste ${ }^{16}$. Cette somme ne comprend pas l'affranchissement des réponses adressées en Russie puisque la plupart des postulants aux études en France envoient des coupons-réponses. Le détail des récépissés indique en revanche une

10. F delta res 815 (11).

11. F delta res 815 (15).

12. F delta res 815 (11), lettre de G. I. Gurevič.

13. Parmi ceux-ci, nombreux, voir : Dmitrij Margolin, Vyščie gornye školy za granicej [Les Écoles supérieures des mines à l'étranger], SPb., 1897 ; Id., Studenčeskij spravočnik [Guide de l'étudiant], t. II : Rukovodstvo dlja postuplenija vo vse vyščie učebnye zavedenija za granicej [Guide d'entrée dans tous les établissements d'enseignement supérieur à l'étranger], Kiev : Samonenko, 1909.

14. F delta res 815 (4).

15. F delta res 815 (10).

16. 261 francs sur un total de 3229 . 
correspondance régulière avec des organes de la presse russe ${ }^{17}$. La plupart des journaux concernés ont une diffusion nationale ou une forte implantation régionale, à Varsovie ou en Ukraine plus particulièrement, et jusqu'en Sibérie. Ils sont de tendance libérale, comme $R e \check{c}^{\prime}$, ou antisémite et réactionnaire, comme Novoe Vremja. L'apolitisme est revendiqué dans une lettre adressée à l'AER par la rédaction du journal Golos Studenčestva (Moscou), bihebdomadaire qui souligne sa neutralité (ne-partijnyj) et se déclare dédié à « servir les intérêts professionnels des étudiants et à les mettre en contact avec les forces vives du pays dans les domaines scientifique et littéraire ». Il propose à l'AER de faire connaître son existence par une annonce payante ${ }^{18}$. Les journaux apparaissent donc comme l'un des principaux canaux d'information pour les candidats au départ, ce que corroborent de nombreux correspondants. Dans l'immense majorité des cas, le courrier révèle le désir, l'impatience même, de partir. Dans une lettre envoyée de Voronež en avril 1911, un jeune homme écrit qu'il a ressenti « une joie indescriptible quand il a appris l'existence de l'AER. » Il souhaite que l'Association l'aide à éviter certaines situations peu favorables dans lesquelles se sont retrouvés ses amis à l'étranger ${ }^{19}$.

Cet étudiant, comme beaucoup d'autres, exprime le soulagement d'avoir trouvé une adresse fiable à Paris où obtenir les renseignements recherchés. L'AER jouit certainement d'une visibilité plus grande que les autres organisations estudiantines russes à l'étranger à tel point que ses responsables reçoivent de nombreuses lettres ayant pour objet les études en Allemagne ou en Suisse. De fait, la plupart des candidats au départ ne font pas la différence entre les pays de la lointaine Europe : ils se rendent là où les conditions d'admission leur semblent les plus simples.

Les étudiants de l'AER formulent des demandes de renseignements par analogie, se représentant la réalité française d'après ce qu'ils connaissent des conditions d'inscription en Russie. Or, le système scolaire russe est très cloisonné, reproduisant par sa rigidité la structure de la société. Seuls les élèves issus des gymnases classiques peuvent s'inscrire à l'université, sans autre limitation que celle imposée aux nationalités (juive en particulier), à la différence de ceux qui ont fréquenté les écoles réales, les écoles de commerce, les écoles techniques et les séminaires, pour lesquels existent, certes, des passerelles, comme les classes complémentaires. De plus, les écoles supérieures d'ingénieurs en Russie, destination normale des diplômés des écoles réales, sont surchargées. Enfin, pour accéder à l'une des neuf universités de l'empire, la connaissance des langues anciennes est nécessaire, d'où les nombreuses questions émanant de correspondants de l'AER sur l'obligation de maîtriser le latin - l'un d'entre eux demande même si en France la médecine est enseignée en latinn ${ }^{20}$. Ce n'est

17. F delta res 815 (3). Odesskie Novosti (Odessa), Novoe Vremja (Saint-Pétersbourg), Golos (Moscou), Russkie Vedomosti (Moscou), Russkoe Bogatstvo (Saint-Pétersbourg), Russkaja Mysl' (Moscou), Reč` (Saint-Pétersbourg), Saratovskie Izvestija (Saratov), Sibirskaja žizn' (Tomsk), Nowa Gazeta (Varsovie), la revue Sovremennik (Saint-Pétersbourg).

18. F delta res 815 (4).

19. F delta res 815 (11), lettre de B. B. Dvorkin.

20. F delta res 815 (11). 
qu'accessoirement que les candidats au départ font état de leur maîtrise insuffisante du français et interrogent l'AER au sujet de cours spéciaux. Pour les responsables de l'AER, une mauvaise connaissance de la langue n'est cependant pas un obstacle insurmontable. Ils encouragent fortement les étudiants à séjourner en France où, en dépit du caractère élitiste de l'enseignement supérieur, l'accès à l'université est plus facile qu'en Russie. Si l'on se fonde sur une réponse de l'AER adressée le 30 mars 1911 à l'un de ses correspondants qui souhaite étudier le droit, l'inscription en France semble plus simple qu'en Russie. On lui conseille l'université de Paris où la durée du cursus est de trois à quatre ans. Il lui faudra présenter une attestation de fin d'études secondaires d'un gymnase classique, d'une école réale ou commerciale ; une fiche d'état civil (metričeskoe svidetel'stvo), puis faire viser ces documents par un consulat de France en Russie. Le ton du responsable de l'AER est enthousiaste. Il conclut sa lettre en encourageant très fortement son correspondant à s'inscrire à l'université de Paris car « la démarche est très simple » 21 .

Néanmoins, la simplicité, toute relative, des démarches en France n'explique pas tout; ce n'est pas dans l'espoir d'obtenir un diplôme sans effort que les étudiants russes décident de s'y installer. La plupart des correspondants de l'AER ont une idée bien précise de la formation qu'ils souhaitent acquérir, contrairement, semble-t-il, aux bacheliers restés en Russie : le professeur N. Kareev indique que ceux-ci choisissent la faculté où ils poursuivront leur cursus souvent au dernier moment, lors des inscriptions 22 .

Le pourcentage de lettres où le cursus envisagé est explicité s'élève à $44 \% 23$. Les étudiants expriment leur préférence pour, par ordre décroissant, la médecine, les sciences (technologie, mathématiques, physique, électrotechnique), l'agronomie et le droit ${ }^{24}$. Les autres filières envisagées, comme les langues (le français), les études musicales, les beaux-arts et le commerce sont, en pourcentage, négligeables. Chez ceux qui ne procèdent pas par analogie et semblent au fait du paysage universitaire français, les questions se font plus précises et portent sur des établissements bien définis tels le conservatoire de musique et d'art dramatique, l'école d'aéronautique, l'École des mines de Paris, l'Institut Pasteur, l'École polytechnique, voire sur des spécialisations pointues, comme l'étude des fibres et des tissus (à Mulhouse, semble-t-il, c'est-à-dire en Allemagne) ou l'œnologie. Mais on trouve aussi des cas d'indécision extrême sur la formation envisagée : géomètre ou vétérinaire, par exemple, ce qui s'explique évidemment lorsqu'il s'agit de demandes collectives.

Les choix et la grande diversité des formations recherchées montrent que la majorité des candidats au départ ont suivi leur scolarité dans des établissements

21. F delta res 815 (8), lettre de l'AER du 31 mars 1911.

22. N. Kareev, Vybor fakul'teta. Rukovodstvo dlja učenikov vyščih klassov srednih učebnyh zavedenij [Le Choix d'une faculté. Guide à l'intention des élèves des classes supérieures des établissements d'enseignement secondaire], SPb., izd. 3, 1897,p. 7.

23. 127 lettres sur un total de 270 .

24. Respectivement $40 \%, 27 \%, 13 \%$ et $9 \%$. 
d'enseignement professionnel - commercial ou technique - ou dans des écoles réales, et optent pour une spécialité fortement recherchée sur le marché du travail russe. Dans le même temps, les étudiants issus des gymnases classiques sont minoritaires parmi les émigrés. En effet, les données que nous avons établies diffèrent notablement des relevés concernant le choix des études en Russie. En 1908, les bacheliers issus de l'enseignement classique se répartissent principalement entre les facultés de médecine, de droit et de physique mathématique ${ }^{25}$. Le professeur Ivanov rappelle qu'une carrière dans le domaine juridique n'est pas envisageable sans un solide réseau de relations. Les étudiants en droit appartiennent généralement à la noblesse ou à la haute bourgeoisie et ne tirent pas avantage professionnellement à s'exiler, ce qui explique leur faible proportion dans nos relevés. En 1908, le nombre de correspondants de l'AER qui demandent des renseignements sur les études de médecine est plus important que celui des élèves issus des lycées classiques et inscrits dans cette même discipline en Russie ${ }^{26}$. Cette disproportion s'explique par le peu de places dans les facultés de médecine russes dont l'accès est limité aux seuls élèves des lycées classiques ou aux étudiants des écoles réales ayant obtenu l' «attestation de maturité »27. La profession de médecin jouissait d'un grand prestige dans les classes moyennes de la société russe et un tel engouement pour les études de médecine s'explique pour différentes raisons. Certes, le diplôme de médecine était la garantie d'une certaine aisance matérielle, mais au-delà de cet aspect financier, les possibilités d'ascension sociale offertes par l'exercice de la médecine s'inscrivent dans une démarche démocratique et humaniste ; tout en gagnant bien sa vie, le médecin reste un homme proche du peuple. Enfin, le choix de ce cursus a sans doute été influencé par la législation en vigueur dans l'Empire russe selon laquelle un diplôme en médecine permettait aux juifs de s'installer en dehors de la zone de résidence, sans devoir se convertir. Nous comprenons mieux pourquoi, parmi les étudiants qui poursuivent des études de médecine à l'étranger se trouvent de nombreux juifs.

Outre ces spécificités russes qui ont pu guider une part des jeunes gens, le souhait d'acquérir un savoir-faire particulier justifie également de poursuivre ses études en France. Ainsi Michel de Bruxelles demande où il peut apprendre l'œnologie et la cuisine, le lieutenant Dorošev cherche une formation dans le domaine de la conserverie ${ }^{28}$, l'ingénieur I. Gurfinkel' d'Odessa souhaite devenir spécialiste du traitement des textiles ${ }^{29}$.

La question de la validation des qualifications acquises en France se situe également au cœur des préoccupations de nombre de correspondants qui ont le souci constant de rentabilité optimale du temps passé à l'étranger. Si certaines filiales d'entre-

25. Ivanov, Studenčestvo Rossii..., p. 34.

26. Environ $30 \%$ pour les années 1908-1909.

27. En 1908-1909, les élèves des écoles réales ne représentaient que 3,3\% de l'ensemble des étudiants en médecine.

28. F delta res 815 (12).

29. F delta res 815 (11). 
prises étrangères en Russie employaient des étudiants russes sur la foi des seuls diplômes obtenus, par exemple, en France ou en Allemagne, le passage devant une commission universitaire russe était souvent nécessaire à la validation des formations.

Sur l'ensemble des lettres, $6 \%$ émanent de jeunes femmes. Il est difficile d'apprécier si ce taux correspond à la proportion de femmes dans la population estudiantine russe en France. Tout en étant inférieur au pourcentage de jeunes filles dans la population estudiantine en Russie ${ }^{30}$, il est sensiblement proche de celui concernant les étudiantes russes à Berlin à la même époque. (Rappelons qu'aucune instance officielle en Russie n'était chargée de relever les chiffres concernant les étudiants russes à l'étranger.)

L'annulation des acquis de la révolution de 1905-1907 entraîne le refus d'admettre les femmes en tant qu'auditrices libres dans les universités. Étudier à l'étranger est donc à nouveau la seule possibilité d'effectuer un cursus équivalent à celui des hommes, même si les chances de voir leurs diplômes reconnus en Russie sont moindres.

Dans la grande majorité des cas, leurs questions ne diffèrent pas de celles des hommes ; tout au plus père ou mère prennent-ils plus facilement la plume en lieu et place de la jeune fille. Elles témoignent du même sérieux, d'une volonté affirmée de se consacrer aux études, ce qui relativise la réputation de débauche et de dépravation des étudiantes de Russie à l'étranger ${ }^{31}$.

Les filières les plus demandées sont, par ordre décroissant, la médecine, les lettres et le droit.

\section{La question nationale}

Près de $10 \%$ des étudiants font part de leur désespoir. Ils se trouvent dans une impasse, ne savent pas quel sens donner à leurs études. Leur courrier est un appel au secours. Nom (juif) et adresse (lieu à communauté juive importante) expliquent cet état. Ainsi M. Barenbojm d'Aleksandrovsk écrit qu'il est prêt à entrer dans n'importe quelle université. Il a fait six ans d'études à Odessa et présente certaines « prédispositions » $\left(\right.$ sic) pour la médecine et les sciences ${ }^{32}$.

Le pourcentage de juifs parmi les étudiants de Russie à l'étranger s'élève, pour la période qui nous intéresse, à environ $80 \% 33$. Il est fort probable que ce pourcentage soit encore supérieur dans certaines villes allemandes. Dans tous les cas, les

30. Ivanov, Studenčestvo Rossii..., p. 136, 177. En 1907-1908 les étudiants étaient plus de 83000 ; entre 1908-1909, les femmes étaient environ 21 000. Durant la période 1913-1914, les chiffres sont respectivement de 121000 pour les hommes et de plus de 27000 pour les femmes. Pour Berlin, voir Weill, Étudiants russes en Allemagne..., p. 125.

31. F delta res 815 (10), lettre envoyée du gouvernement de Smolensk par Ja. S. Belinskij; F delta res 815 (14), celle de S. N. Serpinskij de Majerengof.

32. F delta res 815 (10).

33. Voir, par exemple, V. Karasik, «Russko-evrejskij samizdat v Germanii. Studenčeskij 'Sionistskij listok', Berlin-Mjunhen [Le samizdat russe juif en Allemagne. Le 'Sionistskij Listok', Berlin-Munich] », in Evrei v kul'ture russkogo zarubež ’ja [Les juifs dans la culture de l'émigration russe], Jerusalem, 1998, t. I, p. 23-37. 
juifs représentent le groupe national le plus important de la population estudiantine originaire de l'Empire russe. À cet égard, l'identification de l'origine juive des étudiants est généralement déterminée par l'utilisation de paramètres insuffisamment fiables, tels que l'onomastique et le lieu de résidence. D'autres sources, comme les enquêtes menées par les étudiants eux-mêmes, fournissent des données plus crédibles. Ainsi, en réponse à un questionnaire soumis en 1911 aux étudiants de l'Institut polytechnique de Munich, $68 \%$ indiquent que le système des quotas limitant l'accès des juifs aux universités russes est à l'origine de leur départ à l'étranger ${ }^{34}$. Dans l'ensemble de notre corpus de documents, $30 \%$ des correspondants peuvent être considérés comme juifs. La différence notable avec les pourcentages cités précédemment ( $80 \%$ et $68 \%$ ) s'explique sans doute par le fait que les étudiants, compte tenu de leur appartenance communautaire, s'adressent prioritairement à l'Association des étudiants israélites où, manifestement, certaines personnes parlaient parfaitement russe (dans les archives de l'AER figure une lettre envoyée par les étudiants juifs de France, rédigée dans un russe parfait ${ }^{35}$ ). Pour des raisons linguistiques et culturelles, les juifs choisissent prioritairement de suivre des études en Allemagne. Par rapport aux données chiffrées, les lettres des archives de l'AER apportent un éclairage inédit, humain, sur l'expression de la judéité. Dans certaines, le poids de la souffrance est bien perceptible, dans d'autres, l'appartenance est à peine évoquée, comme si elle n'était qu'un facteur secondaire. Tout porte à croire que, pour ces étudiants juifs, le choix d'étudier à l'étranger s'inscrit, à l'instar des jeunes Russes non juifs, dans le cadre général de l'acquisition d'une formation professionnelle. Les jeunes juifs ont intégré l'idée d'un départ nécessaire en Europe après le lycée classique ou l'école réale.

La lecture des lettres d'expéditeurs juifs donne un reflet diversifié et vivant de la situation des étudiants juifs et de leurs motivations à quitter l'Empire russe.

MM. Gerškovič de Kagul’ en Bessarabie ${ }^{36}$ et Aleksandr Rišman de Vajnoden en Courlande ${ }^{37}$ font à peine allusion à leur judéité. Le second écrit qu'en Russie, il est privé de la possibilité de poursuivre ses études. Un père de famille, Ja. S. Belinskij de Dukovščina dans le gouvernement de Smolensk, en revanche, indique clairement que sa fille est obligée de partir à l'étranger parce qu'elle est juive ${ }^{38}$.

I. Bialik et Izrail Kuržnir du gouvernement de $\mathrm{Kiev}^{39}$, F. Gercberg d'Odessa ${ }^{40}$, et M. Jakovlevič Hodzin de Tomsk ${ }^{41}$ expliquent, eux aussi, que l'accès à l'université leur est fermé parce qu'ils sont juifs. David Srulevič Fridman est dans le même cas. Il

34. Ivanov, Studenčestvo Rossii..., p. 349.

35. F delta res 815 (7).

36. F delta res 815 (11).

37. F delta res 815 (14).

38. F delta res 815 (10).

39. F delta res $815(10),(11)$.

40. F delta res 815 (11).

41. F delta res 815 (15). 
précise cependant qu'en dépit des difficultés, il considère l'Empire russe comme sa « patrie » ${ }^{42}$. Ces exemples attestent bien que le départ à l'étranger peut être qualifié d' « opportunité contrainte », selon l'expression de Victor Karady ${ }^{43}$. Certains jeunes gens, comme M. Hodzin ${ }^{44}$ et M. Gerškovič ${ }^{45}$, affirment leur appartenance juive avec fierté. Ils souhaitent partir en France pour ne pas avoir à payer le droit d'entrée à l'université au prix du reniement de leur culture et de leur religion.

\section{Les raisons politiques et la question du service militaire}

D'autres étudiants expriment le désir de quitter la Russie parce qu'ils y sont considérés comme dangereux en raison de leurs activités révolutionnaires et ne peuvent donc s'inscrire à l'université. Aucun correspondant ne précise cependant les raisons de son exclusion, pas plus Aleksandr Mihajlovič46 Lozin de Bugrovskij en Crimée, qui a étudié la chimie à l'université de Kharkov, que S. L. Radomyšelskij ${ }^{47}$ du gouvernement de Poltava, exclu après deux ans d'études à la faculté de mathématiques et de physique. A contrario, c'est la situation chaotique dans les universités, en réponse aux mesures restrictives, qui empêche la poursuite sereine des études, comme ce fut déjà le cas lors de la révolution de 1905. «Grâce (sic) à la grève actuelle, mes espoirs d'achever l'institut polytechnique de Kiev ont toutes les chances de s'écrouler » écrit Granat de Kiev le 6 mars $1911^{48}$.

L'appel sous les drapeaux à l'âge de 21 ans, selon la loi du 1er janvier 1874, contraignait les redoublants à interrompre leurs études ainsi que ceux qui avaient échoué au concours d'entrée à un institut spécialisé. Les étudiants des instituts ou des facultés dont la durée des études était de quatre ans pouvaient obtenir un sursis jusqu'à 27 ans, jusqu'à 28 ans pour les cycles de cinq ans. C'est dans ce domaine que l'urgence apparaît le plus nettement, certains correspondants demandant même de les inscrire avant leur venue en France afin qu'ils obtiennent l'attestation qui leur ouvrira la voie du sursis. La lettre d'Evsej Borisovič Sigal de Tul'čin du 4 mai 1911 est révélatrice d'un cercle vicieux. Il est appelé sous les drapeaux à partir du 4 novembre 1911. Pour obtenir un sursis, il lui faut s'inscrire dans une université à l'étranger. Il a donc besoin d'un passeport, qu'il n'obtiendra qu'avec l'attestation d'inscription dans un établissement d'enseignement supérieur à l'étranger où, cependant, il ne peut se rendre sans passeport... ${ }^{49}$ Les nombreuses questions posées

42. Ibid.

43. Karady, Relations inter-universitaires...,p. 520.

44. F delta res 815 (15).

45. F delta res 815 (11).

46. F delta res 815 (13).

47. F delta res 815 (14).

48. F delta res 815 (11).

49. F delta res 815 (14). 
à l'AER ne permettent pas d'apprécier si ce type d'inscription était un moyen d'échapper au service militaire ou si les postulants cherchaient simplement à bénéficier des mêmes mesures qu'en Russie.

Le dernier groupe de correspondants est constitué de cas « marginaux ». Certains d'entre eux évoquent des raisons de santé50 ${ }^{0}$ d'autres, généralement des couples ou des soldats, expriment le souhait de s'installer définitivement en France ${ }^{51}$.

\section{Les conditions de vie}

Les hésitations entre établissements parisiens ou provinciaux se situent à la charnière des interrogations sur les cursus possibles et les conditions de vie en France. Elles sont souvent levées au profit de la province et cela, moins en raison de la qualité de l'enseignement que du coût de la vie qui y est supposé inférieur - motif qui incite un étudiant à envisager une installation en Algérie ${ }^{52}$. En second lieu, c'est la perspective d'une formation spécifique qui attire les étudiants de Russie en province. Enfin, le climat, la qualité des relations avec les enseignants entrent également en ligne de compte. Les villes le plus souvent citées sont Nancy, Montpellier, Toulouse et Grenoble.

Dans l'ensemble, si les jeunes femmes se montrent plus préoccupées par les conditions matérielles, les difficultés financières n'épargnent guère leurs compatriotes masculins. Le courrier en provenance de Russie illustre la faiblesse des ressources pécuniaires de la plupart des postulants à la migration étudiante : B. Sigal demande s'il est plus avantageux d'étudier à Paris ou dans une autre ville ${ }^{53}$, une jeune fille, L. I. Hižickaja, veut savoir s'il est possible de vivre en province avec trente roubles mensuels, V. N. Panov de Tiflis calcule son budget au plus près : «Je voudrais savoir [...] quelle est la somme approximative nécessaire pour vivre, si l'on se prive de loisirs comme des sorties au théâtre, au café ${ }^{54}$, enfin, Izrail’ Kuržnir, originaire de la bourgade juive de Zlatopol, précise qu'il ne dispose que d'un budget de misère de vingt-quatre roubles par mois. Ce dernier n'a donc pas d'autre solution que de partir à l'étranger et se déclare prêt à donner des leçons pour gagner un peu d'argent ${ }^{55}$. Ces lettres révèlent le bas niveau social de la majorité des étudiants russes et l'espoir de ceux-ci de trouver un coût de la vie moins élevé qu'en Russie. Enfin, de nombreuses questions ont trait au prix du voyage et surtout au montant des inscriptions.

50. F delta res 815 (10), lettre envoyée depuis Bad Nauheim ; F delta res 815 (14), Vitali Smirnoff cherche une ville au climat chaud; F delta res 815 (15), D. Fisch de Davos.

51. F delta res 815 (15), lettre d'Otto Ejzenberg; F delta res 815 (13), lettre de Valdemar Mirsky et Michael Preobraschensky (sic); F delta res 815 (15), soldat Aseev.

52. F delta res 815 (14).

53. Ibid.

54. F delta res 815 (13).

55. F delta res 815 (11). 
Les questions peuvent être aussi d'une précision redoutable. Ainsi Nikolaj Mihajlovič Guržeev de Saint-Pétersbourg voudrait connaître le prix d'un appartement de deux-trois pièces à Paris, non meublé, modeste, mais dans une partie « saine » de la ville et demande si les locations se font au mois ou à l'année. Il s'enquiert aussi du coût d'une femme de ménage, du prix du lait entier, de la viande de bœuf, de veau, de mouton, du poulet, du poisson, d'une dizaine d'œufs et du beurre de lait de vache non pasteurisé56. Ces demandes émanent manifestement d'un jeune homme disposant de revenus au-dessus de la moyenne et soucieux de la qualité de la nourriture. C'est le cas aussi de cette mère qui veut inscrire son fils au conservatoire de musique et souhaite habiter Passy. Elle aimerait aussi savoir si elle-même peut gagner sa vie en donnant des concerts ${ }^{57}$. Mademoiselle Gadzjaukaja de Grodno est manifestement une étudiante très aisée. Elle demande si avec six cents roubles il est possible de louer rive gauche au $2^{\mathrm{e}}$ étage (c'est-à-dire au premier, d'après le système français) un appartement de trois pièces, cuisine, avec garage ${ }^{58}$.

\section{Fonctionnement interne de l'association}

L'AER apportait une aide morale et matérielle aux étudiants. Des lieux comme la bibliothèque ou le restaurant, dont elle avait la gestion, favorisaient le maintien et le développement de liens de sociabilité entre les émigrés.

\section{Gestion de l'organisation}

Les deux fonds, ceux de l'AER et les archives Bebutov, contiennent plusieurs bilans financiers qui permettent de comprendre le fonctionnement de l'Association ${ }^{59}$. La diversité des postes budgétaires atteste du caractère autonome de l'AER qui offrait aux étudiants russes le même type de services que les organisations estudiantines équivalentes en Russie ${ }^{60}$. Les bénéfices provenant du bal de fin d'année ainsi que les cotisations permettaient en tout premier lieu d'apporter une aide financière sous forme de crédits courts d'une durée maximum de trois mois destinés à couvrir les frais de scolarité, les loyers et les soins médicaux ; des crédits longs, pour la durée des études, étaient accessibles aux étudiants inscrits dans des établissements d'enseignement supérieur. Les demandes de prêt nous renseignent en

\section{F delta res 815 (11).}

57. F delta res 815 (15), lettre de madame S. F. Čužbonskaja.

58. F delta res 815 (11).

59. F delta res 815 (1), pour les années 1908, 1909, 1910 ; F delta res 811 (8), (12), pour les années 1913, 1914.

60. A. Ivanov, Studenčeskaja korporacija Rossii konca 19-načala 20 veka : opyt kul'turnoj $i$ političeskoj samoorganizacii [La corporation des étudiants en Russie à la fin du XIXe et au début du XXe siècle : essai d'autogestion culturelle et politique], M.: Novyj hronograf, 2004. 
creux sur les frais d'inscription et les prix de location, du moins à Paris. L'AER estime d'ailleurs de cent à cent vingt francs le budget mensuel moyen nécessaire, soit environ trente-cinq à quarante-trois roubles ${ }^{61}$. Les nombreux « appels à l'aide » montrent que, dans l'ensemble, les budgets des étudiants russes étaient souvent limités, voire insuffisants ${ }^{62}$. Les recours au crédit étaient particulièrement fréquents en mai et en juin, lors des inscriptions payantes aux examens.

Afin de disposer des moyens nécessaires à leur séjour en France, de nombreux étudiants exerçaient une activité rémunérée. Au sein de l'AER un bureau du travail proposait des emplois-service ${ }^{63}$. Les sphères d'activité (garde-malade, répétiteur, guide, etc.) limitaient les contacts à l'émigration russe. Cette volonté de rester «entre soi » se manifeste dans de nombreuses lettres envoyées depuis la Russie. Des correspondants demandent des adresses de personnes originaires de Russie et résidant à Nancy, Lyon et même en Algérie ${ }^{64}$.

\section{Le restaurant universitaire}

Le restaurant universitaire était un peu comme une enclave russe. Les étudiants y retrouvaient leurs compatriotes. Ils pouvaient aussi s'y nourrir à des prix plus abordables que dans d'autres lieux de restauration.

Au dos d'un menu en français conservé dans les archives de l'AER figure une liste de questions inscrites à l'ordre du jour d'une réunion concernant l'organisation du restaurant situé 40, rue Saint-Jacques à Paris : renouvellement par un vote démocratique du comité de direction composé de vingt-cinq membres ; établissement de relations de respect et de compréhension mutuelles entre les responsables de la gestion, les cuisiniers et le personnel de salle. Les interactions entre ces différentes catégories du personnel sont représentées par plusieurs schémas visant à améliorer le fonctionnement démocratique des structures en place.

Dans un coin du document, une petite note attire l'attention : « aborder la question des claques », ce qui laisse à penser que les débats politiques au restaurant pouvaient parfois dégénérer en rixes.

Le professeur A. Ivanov, spécialiste de l'histoire de la vie étudiante en Russie au $\mathrm{XIX}^{\mathrm{e}}$ et $\mathrm{XX}^{\mathrm{e}}$ siècle, précise que les renseignements sur la manière dont les étudiants russes se nourrissaient à l'étranger sont très limités. Les sources sont certes parci-

61. F delta res $815(8)$.

62. F delta res 815 (10), lettre de D. Bortman: F delta res 815 (12), lettre de Grinberg, O. Zejberg ; F delta res 815 (13), de Madame Primiak ; F delta res 815 (14), de B. Sikorov ; F delta res 815 (15), Mme Endrikson écrit de Zurich.

63. F delta res 815 (3).

64. F delta res 815 (12), Ju. Jolis, de Berdičev ; F delta res 815 (14), Pavel Eduardovič Rustejko de Saint-Pétersbourg et Klavdija Pavlovna Kotova de Krasnolobodosk ; F delta res 815 (15), L. I. Hižinskaja de Sudilkovo, gouvernement de Volhynie. 
monieuses, néanmoins, une réclame trouvée dans les archives de l'AER témoigne d'un très grand soin apporté à la qualité des produits ${ }^{65}$.

\section{La bibliothèque}

Avec le restaurant et le bureau du travail, la bibliothèque servait de lieu de ralliement pour les émigrés.

La salle de lecture de l'AER, fondée en 1908, la même année que le restaurant, fut appelée, sans grande originalité, bibliothèque Léon Tolstoï ${ }^{66}$. À cette époque, Paris comptait plusieurs bibliothèques « russes » qui dépendaient d'associations nationales ou politiques : la bibliothèque juive Pernikov (5, avenue de la République, $11^{\mathrm{e}}$ arrondissement), le Club des juifs russes de Paris (65, rue de la Concorde, 19e arr.), la bibliothèque de l'Union démocratique républicaine (18, rue de Varenne, $7 \mathrm{e}$ arr.), le Mouvement des étudiants chrétiens russes (10, boulevard du Montparnasse, $15^{\mathrm{e}}$ arr.), la bibliothèque des socialistes révolutionnaires (SR) (81, rue Pascal, $5^{\mathrm{e}}$ arr.), la bibliothèque polonaise Mickiewicz (6, quai d'Orléans, $4^{\mathrm{e}}$ arr.), la bibliothèque de la social-démocratie (63, avenue des Gobelins, 13e arr.), et la plus célèbre, la bibliothèque Tourgueniev (328, rue Saint-Jacques, $5^{\mathrm{e}}$ arr.)

La bibliothèque de l'AER offrait une grande variété de journaux de différentes sensibilités politiques, signe du caractère « politiquement neutre » de l'association. Les étudiants pouvaient y consulter: Odesskie Novosti, Novoe Vremja, Golos Moskvy, Sovremennyj Peterburg, Russkie Vedomosti, Russkoe Bogatstvo, Russkaja Mysl', Reč', Rus', Birževye Vedomosti, Russkoe Slovo, Sovremennyj Mir, Mir Božij, Obrazovanie, Šipovnik, Vestnik Znanija, Russkij Vrač, Novoe v medicine, Medicinskoe Obozrenie, Kievskaja Mysl', Har'kovskoe Utro, Saratovskie Izvestija, Le Petit Quotidien, Le Petit Journal...

Les règles de fonctionnement de la bibliothèque étaient affichées à l'entrée du local : seuls les membres de l'AER avaient le droit de fréquenter la salle de lecture ; chaque visiteur devait émarger dans un cahier disposé à cet effet. Signatures, journaux lus et livres empruntés permettaient de tracer le « profil type » des lecteurs.

La multiplication des bibliothèques ne favorisait pas un fonctionnement efficace. De surcroît, les moyens financiers de chacune étaient insuffisants pour offrir aux lecteurs tous les ouvrages qu'ils désiraient. Pour pallier ces manquements, les responsables de l'AER comprirent rapidement la nécessité de s'associer à une autre salle de lecture. En 1910, des contacts furent établis avec la bibliothèque Tourgueniev. L'AER avait conçu le projet de fédérer l'ensemble des organisations russes de Paris et de constituer une Maison russe (Russkij Dom). Ce lieu aurait eu vocation à devenir un centre culturel où les étudiants auraient suivi gratuitement des leçons de botanique, de zoologie et de physique ainsi que des cours de perfectionnement en

65. F delta res 815 (5).

66. De nombreuses bibliothèques russes à l'étranger portaient le nom de l'illustre écrivain. Voir Weill, Étudiants russes en Allemagne... 
français $^{67}$, mais ce projet n'aboutit pas. Dans un courrier, les responsables de la bibliothèque Tourgueniev expliquent qu'ils sont d'accord pour transférer leurs ouvrages dans un nouveau local, à condition d'en conserver le contrôle et que le coût du déménagement soit pris en charge par l'AER ${ }^{68}$. Les pourparlers n'allèrent pas au-delà de l'évocation d'un projet commun.

Cette tentative illustre la tendance à l'unification des organisations russes qui se manifesta dans les années 1910. Ainsi, au moment où l'AER et la bibliothèque Tourgueniev entraient en contact, le Comité des étudiants russes à Lausanne proposait à l'AER de constituer un centre de documentation unique où seraient entreposés tous les documents concernant la vie des étudiants russes en Europe ${ }^{69}$.

\section{Les enquêtes}

Afin d'optimiser la qualité de ses services et de définir les caractéristiques des personnes qui s'adressent à elle, l'Association mène en 1909 une enquête auprès des étudiants russes. La pratique du questionnaire (samoperepis') est un des phénomènes les plus originaux de la vie estudiantine russe de la période d'avant la révolution de 1917. À notre connaissance, les universitaires et les élèves des grandes écoles russes sont les seuls en Europe à organiser des sondages dont l'objectif est la constitution d'un portrait type d'étudiant. Ainsi dans l'introduction du samoperepis' mené en 1915 par les auditrices de l'Institut Bestužev, le professeur A. A. Kaufman indique que le but premier en est d' " éclairer la situation matérielle des étudiantes à la lumière impartiale des chiffres »; il s'agit d'un projet scientifique qui a pour finalité de présenter à la société russe qui sont « réellement les étudiant(e)s $»^{70}$. À peu près à la même époque, le docteur S. P. Nikol'skij argumente :

Il existe des renseignements contradictoires et souvent faux sur les conditions de vie de nos étudiants et de fait, l'idée que l'opinion publique a de nos élèves ne correspond pas à la réalité [...] La société ne sait pas où ni comment vivent nos étudiants, quelles sont leurs conditions de vie. On ignore également comment ils se nourrissent, ce qu'ils boivent, en quelle quantité, combien de tabac ils consomment et de quelles maladies ils souffrent ${ }^{71}$.

\footnotetext{
67. F delta res $815(8)$.

68. F delta res $815(7)$.

69. Ibid.
}

70. A. A. Kaufman, éd., Slušatel'nicy Petrogradskih vyščih ženskih (Bestuževskih) kursov na vtorom godu vojny: bjudžet, žiliščnye uslovija, pitanie. Po dannym perepisi, vypolnenoj statističeskim seminariem v konce oktjabrja $1915 \mathrm{~g}$. [Les Auditrices des cours supérieurs de Petrograd (cours Bestužev) pendant la deuxième année de guerre : budget, conditions de logement et de nourriture. D'après les données du recensement réalisé par le séminaire de statistique à la fin du mois d'octobre 1915], M., 1916, p. 111.

71. S. P. Nikol'skij, K harakteristike sovremennogo studenčestva : po dannym perepisi 190910 gg. v S-Peterburgskom tehnologičeskom institute [Vers une caractérisation des étudiants : d'après les données du recensement de 1909-1910 à l'Institut technologique de SaintPétersbourg], SPb., 1911,p. 71. 
De 1872, date de la première enquête ${ }^{72}$, jusqu'en 1917, les samoperipisi sont réalisés par les étudiants eux-mêmes, avec parfois l'aide de professeurs d'économie et de statistiques ${ }^{73}$. Une telle indépendance illustre le peu de crédit que les étudiants accordent aux autorités administratives et politiques. Ils craignent que certaines données ne soient utilisées par le pouvoir. Le passage à l'université est pour les jeunes Russes une période d'apprentissage de la vie sociale et démocratique ; l'organisation d'enquêtes en fait partie.

Le contenu des questionnaires évolue avec le temps. Les six enquêtes menées entre 1892 et 1898 comportaient un nombre restreint de questions qui concernaient essentiellement les conditions matérielles. Entre 1900 et 1917, elles portent sur des sujets aussi variés et précis que les pratiques sexuelles ou l'engagement politique. L'intérêt croissant pour les critères de définition de l'identité nationale se concrétisa, entre 1909 et 1913 à Kiev, par l'organisation de samoperepisi parmi les étudiants juifs $^{74}$. En Russie le recours aux questionnaires était relativement fréquent, alors qu'à l'étranger leur nombre fut limité. Le professeur A. Ivanov se réfère à deux samoperepisi réalisés respectivement à Grenoble en 1910 et à Munich en $1914^{75}$. Claudie Weill évoque une autre enquête effectuée en 1914 qui comportait 37 questions ${ }^{76}$.

Le fonds Bebutov contient des formulaires vierges d'un sondage effectué en 1909 en France, résultat d'une collaboration étroite entre l'AER, la bibliothèque Tourgueniev, les socio-démocrates (SD), les socialistes révolutionnaires (SR), la bibliothèque polonaise Mickiewicz et des établissements russes de restauration universitaire ${ }^{77}$. La diversité des commanditaires montre qu'en dépit des antagonismes d'ordre national ou politique les organisations « russes » maintenaient entre elles d'étroites relations. L'enquête permettait de mieux cerner les caractéristiques des émigrants. Elle favorisait également le rapprochement entre des étudiants appartenant à des groupes nationaux ou politiques distincts.

L'enquête dont nous disposons comprend 61 questions. Elle rentre par conséquent dans la catégorie des questionnaires dits complexes (kompleksnye) destinés à

72. La première enquête fut réalisée à Kiev en 1872 à l'initiative du futur ministre des Finances, le professeur N.H. Bunge: V.V.Svjatlovskij, Studenčeskie perepisi V Rossii. Kratkij istoričeskij očerk : studenčestvo v cifrah. Po dannym perepisi 1907 v Jur'eve [Recensement d'étudiants en Russie. Essai historique : le monde étudiant en chiffres d'après les données du recensement de 1907 à Jur'ev], SPb., 1907, p. 5.

73. T. Petrova, Sociologija studenčestva $v$ Rossii. Etapy i zakonomernosti stanovlenija Sociologie du monde étudiant], SPb., 2000.

74. D. Šejnis, Evrejskoe studenčestvo v cifrah. Po dannym perepisi $1909 \mathrm{~g}$ v kievskom universitete i politehničeskom fakul'tete [Les étudiants juifs en chiffres. D’après les données du recensement de 1909 à l'université polytechnique de Kiev], Kiev, 1911; Evrejskoe studenčestvo v Moskve. Po dannym ankety $1913 \mathrm{~g}$. [Les étudiants juifs à Moscou. D'après les résultats de l'enquête de 1913], M., 1913; K harakteristike evrejskogo studenčestva. Po dannym ankety sredi evrejskogo studenčestva g. Kieva [Vers une caractérisation des étudiants juifs d'après les données de l'enquête menée à Kiev auprès des étudiants juifs], Kiev, 1913.

75. Ivanov, Studenčeskaja korporacija..., p. 120-122.

76. Claudie Weill, «Convivialité et sociabilité des étudiants en Allemagne, 1900-1914 », Cahiers du Monde russe et soviétique, 32 (3), juillet-septembre 1991, p. 349-368.

77. F delta res 811 (8), (12). 
donner une photographie très large du monde estudiantin russe en France. Nous ignorons combien de personnes y répondirent et les conclusions qui en furent tirées. Il nous faut donc nous «contenter» des questions sur les principaux aspects de la vie des étudiants, que les organisateurs du sondage souhaitaient éclairer.

Les questions les plus nombreuses ont trait aux conditions de vie et, plus précisément, à la situation financière : montant et répartition des dépenses, sources de financement des études, logement et loisirs (café, théâtre). Un second groupe de questions concerne le niveau d'études, les formations recherchées et la durée envisagée du séjour en France. Le questionnaire aborde aussi la vie personnelle et, en particulier, les caractéristiques nationales et politiques. Les questions sur l'origine géographique, les pogroms, les langues parlées, servaient manifestement à déterminer le pourcentage d'étudiants juifs et les raisons de leur départ à l'étranger. D'autres rubriques posent directement la question de l'appartenance à un parti politique russe et de la nature des relations politiques ou simplement amicales avec les Français. Ce dernier point est à rapprocher des questions sur le degré d'intégration dans la société française : lectures, loisirs, sorties. Enfin, un petit nombre de questions concerne le fonctionnement et la visibilité de l'AER.

\section{Conclusion}

De toute évidence, la politique des quotas limitant l'accès des juifs aux universités russes est une des principales raisons des départs à l'étranger. Les documents de l'AER permettent toutefois de nuancer cette image. Ils illustrent en particulier le retard du système d'enseignement en Russie, qui n'offrait pas les formations professionnelles auxquelles aspiraient les premières générations de la révolution industrielle. Partir à l'étranger était souvent une nécessité politique et nationale, mais entre 1909 et 1914 cela devint un phénomène de masse et une démarche presque banale dans le cursus d'un étudiant de l'Empire russe.

À l'étranger, les différences politiques et nationales subsistaient. L'étude des documents de l'AER illustre pourtant la maturité sociale des étudiants russes dans la gestion des organisations estudiantines. À de nombreux égards, ils se substituaient dans ce domaine au régime tsariste défaillant et incarnaient l'avenir démocratique de la Russie. Néanmoins, pour un grand nombre d'entre eux, le séjour en France ou en Allemagne ne s'inscrivait pas dans une perspective politique, mais uniquement académique. En 1917, seuls quelques-uns se retrouvèrent sur les barricades, les armes à la main et, en 1920, ceux qui avaient poursuivi leurs études avant la Révolution étaient qualifiés péjorativement de « vieux » représentants de la société bourgeoise ${ }^{78}$.

\section{BCzerny@aol.com}

Université de Caen, département d'études slaves

78. A. Markov, Čto značit byt' studentom [Ce que signifie être étudiant], M.: Novoe literaturnoe obozrenie, 2005. 